\title{
Truth Statements in Denial Context: A Study of Prosodic, Paralinguistic, and Discursive Cues in Japanese Speakers
}

Anna Danielewicz-Betz* and Naomi Ogasawara

Centre for Language Research, University of Aizu, Tsuruga 90, Ikki-machi, Aizuwakamatsu, Fukushima 965-8580, Japan

\begin{abstract}
This study investigates linguistic and paralinguistic characteristics of truth statements in denial contexts in terms of prosodic and discursive cues exhibited by Japanese speakers. The majority of studies to date have either focused on lie detection or distinction between true and false statements. Moreover, the subjects have been mostly been Caucasian English-speakers. Observers also tend to resort to stereotypical preconceptions related to liars behaviour, failing to note how truth tellers behave.

We conducted an experiment with native Japanese speakers from the Tohoku area (north-east part of Japan) who are perceived as more introvert, patient, and less outgoing in comparison with those from other regions of Japan Eleven undergraduate students of both genders were recruited and interviewed individually, with their answers recorded and videotaped. The subjects faced some false statements related to their activities or whereabouts on a particular occasion. Knowing that those statements were all false, it was expected for the subjects to deny them, trying to convince the interviewer of their telling the truth and the interviewer's mistaken claims. Their utterances were examined with respect to three main parameters: prosody (pitch change, stress, pauses), non-verbal behaviour (e. g. gaze, mimics, body part movements), and discourse cues (lexical, syntactic, pragmatic). A content and quantitative analysis of the strategies employed in false claim denials and truth verification was then carried out.
\end{abstract}

Keywords: Forensic linguistics; Japanese culture; Truth-telling cues; Negation and denial; Acoustic analysis; Conversation analysis; Pragmatics

\section{Introduction}

Forensic linguistics is an interdisciplinary field of applied/ descriptive linguistics that comprises the study, analysis and measurement of language in the context of crime, judicial procedures, or disputes in law. The interface between language, crime and the law can be detected, for instance, in the analysis of courtroom discourse, courtroom interpreting and translating, the comprehensibility of legal documents, the comprehensibility of the police caution issued to suspects, and authorship attribution. Forensic linguistics uses the expertise of descriptive and applied linguists in the unraveling of legal puzzles, so to say. Informed use of forensic linguistics requires familiarity with the broader application of linguistics as a social science, including phonetics and phonology, morphology, syntax, and semantics, discourse analysis, pragmatics, psycholinguistics, neurolinguistics, sociolinguistics, dialectology, computational linguistics, and corpus linguistics.

The forensic linguist applies linguistic knowledge and techniques to the language implicated in legal cases or proceedings and private disputes between parties which may result in legal action. Restrictions in applying linguistic expertise in the context of law are due to varying degrees of acceptability of the evidence provided in the courtroom; varying degrees of reliability related to shortcomings such as the brevity of documents, small data samples, general characteristics of language (for example, generic language features of suspects), and the intrinsic nature of language in constant change. The quality of evidence from this emerging field also depends considerably on the experience and knowledge of individual linguists involved in a given case. Courts in many countries admit forensic evidence but have differing criteria. In the United States, for example, the so-called Daubert standard rule of evidence regarding the admissibility of expert witnesses' testimony in federal legal proceedings states that evidence based on innovative or unusual scientific knowledge may only be admitted after it has been established that it is reliable and scientifically valid. The Daubert test is based on peer review, error rates, testing, and acceptability in the relevant scientific community.

This study investigates the linguistic and paralinguistic characteristics of truth statements in denial contexts. The majority of studies have either focused on lie detection or distinction between true and false statements [1-3], failing to note how truth tellers behave [4-5]. Moreover, the subjects have been mostly Caucasian English-speakers [6]. By contrast, we focus on prosodic and discursive cues exhibited by Japanese speakers born in the north-eastern part of Japan, who are commonly perceived as more introvert, patient, obstinate, and ingroup-oriented in comparison with those from other regions [7].

As Vrij [6] states, it could be that people of different ethnicities or cultures show different non-verbal cues to deceit (give-away cues). Unfortunately, there is not much cross-cultural deception research. Most deception studies have been carried out in Western countries where the vast majority of participants were of Caucasian origin. In other words, the research findings published to date tell us how Caucasian liars behave (15\% loc 1712, Kindle version). No differences between ethnic groups were found in Sitton and Griffin [8], nor in Vrij

*Corresponding author: Dr. Anna Danielewicz-Betz, Centre for Language Research, University of Aizu Tsuruga 90, Ikki-machi, Aizuwakamatsu, Fukushima 965-8580, Japan; Tel: +81 0 242-37-2586, Fax: +81 0 242-37-2599; E-mail: adanielewicz_betz@yahoo.com

Received November 27, 2012; Accepted December 27, 2012; Published December 29, 2012

Citation: Danielewicz-Betz A, Ogasawara N (2013) Truth Statements in Denia Context: A Study of Prosodic, Paralinguistic, and Discursive Cues in Japanese Speakers. J Forensic Res S11: 001. doi:10.4172/2157-7145.S11-001

Copyright: (c) 2013 Danielewicz-Betz A, et al. This is an open-access article distributed under the terms of the Creative Commons Attribution License, which permits unrestricted use, distribution, and reproduction in any medium, provided the original author and source are credited. 
and Winkel [9]. Regarding individual differences, as far as we know, there have been no studies carried out examining gender or ethnic (cultural) differences in individual verbal cues to deception ([6] $21 \%$ loc 2424, Kindle version).

\section{The Japanese Cultural Context}

According to Hofstede [10], Japan falls into 'high on collectivism' cultural category (this value indicates how closely a society is knit). In collectivist cultures the needs, values and goals of the family and societal unit take precedence over individual goals. Group consciousness and family are the major values that guide people's behaviour. It is also a high power distance culture that accepts power and hierarchy in society and is low on egalitarianism. This indicates that less powerful citizens accept unequal power distribution in society. Japan is also in the high uncertainty avoidance category. This means importance of predictability, structure and order, and unwillingness to take risk. People from cultures high on uncertainty avoidance like Japan tend to have low tolerance for uncertainty. They avoid ambiguous situations, view both conflict and competition as threatening, and value security over adventure and risk. On the masculinity-femininity scale, whereby a belief in achievement and ambition is categorised as 'masculine' with a belief in nurturing and caring for others being perceived as 'feminine', Japan scores as an extremely masculine culture. Moreover, high context cultures like Japan exhibit close connections among group members. Everyone has a similar, intrinsic knowledge base. In addition, they use more symbols and non-verbal cues to communicate.

The concept of aimai (あいまい), i.e. “ambiguity”, is one of the key elements of traditional Japanese society and relates directly to maintenance of harmony or wa (わ) in tight communities. People tend to avoid expressing their ideas clearly, even to the point of not giving a simple yes or no answer. To signal a no, one may say nothing at first, and then used vague expressions that convey the nuance of disagreement [11].

So in the high-context Japanese culture things are not clearly and directly spelt out, leaving it up to the listener to interpret the utterance the way she/he wishes. In general there is little said; conversations are left incomplete, with the rest implied by the context. Therefore a direct denial would be rare and a non-response may be interpreted as a denial.

Symbols and non-verbal cues are used to communicate, with meanings embedded in the situational context. Constant guessing is performed about how others might feel or what they might want. Due to the lack of ethnic diversity - sharing the same language and customs by the whole population - the interpretation of the unsaid, indirect, or unfinished comes in relatively easy and thinking about other people's needs first seems natural [12].

Turning to conflict avoidance as demonstrated in conversation, disagreement is commonly delayed and mitigated [13], which will be shown in the discussion of our data. As long as both the speaker and the listener are able to detect the meaning between the lines, especially when criticising or rejecting people, i.e. hurting other people's feeling in some way, no clear expression of opinion is necessary. Thus, "hai" in Japanese means something like "what you are saying is right" or "it's correct", and "lie" means more or less "what you are saying is not right" or "it's not correct".

This is an example from our data (audio transcription, literal translation, file 0206, female):

Q: Weren 't you in the store in those clothes.

A: No, I wasn't there.

\section{Q: Really?}

A: Yes.

Q: I think I met you.

A: Hmm. I was at home last Saturday.

Q: Were you at home all the time? Didn't you visit your friends?

A: Yes.

Generally speaking, in an affirmative question:

"Hai" in Japanese = "Yes" in English

"lie" in Japanese = "No" in English

But in a negative question:

"Hai" in Japanese = "No" in English

"lie" in Japanese = "Yes" in English [14]

\section{Methods, Research questions, and Limitations of the Study}

In order to investigate (para-) linguistic characteristics in truthful statements by Japanese speakers, we conducted an experiment for which eleven undergraduate students from the University of Aizu were recruited. We selected subjects who were born and raised in the Tohoku (north-eastern) region of Japan, where people are generally perceived as introverted, patient, and not very articulate.

The experiment was carried out in two phases: a questionnaire and a semi-structured interview. First, the subjects filled in a behavioural questionnaire that was designed to detect their personality traits of lying. We shortened and modified the questions used by Abeler et al. [15]. The questionnaire was designed to elicit some personal information regarding the place of origin, interests, and the like, and to aim at self-assessment of non-verbal behaviour, body language, and personal characteristics related to embarrassing and stressful situations, self-esteem, keeping secrets, and lying. The 16 statements on the questionnaire were rated on a 1 to 7 scale (1: absolutely disagree/not OK to do under any circumstances, 2: strongly disagree/not acceptable, 3: disagree/slightly not acceptable, 4 : neither, 5 : agree/slightly acceptable, 6: strongly agree/acceptable, and 7: absolutely agree/definitely $\mathrm{OK})$. The instructions and statements were all written in Japanese (see Appendix 1 for the English version of the questionnaire).

In Phase 2, two senior students who also spoke a dialect close to the subjects' dialects interviewed the subjects individually. The interviews took place in a sound-attenuated chamber in the University of Aizu. Each subject was seated in front of the interviewers across a small table. Prior to the interview session, the interviewers were trained by a Japanese experimenter (one of the authors), and instructed to follow the same course of the interview for each subject: 1) small talk as an icebreaker-asking about the name, age, hometown, club activities, and blood-type; 2) doubting the subjects' telling the truth about their hometown; and 3) claiming that one of the interviewers happened to see the subject at a convenience store near the local train station last Saturday morning. The interviews took 7 to 10 minutes. They were all video- and audio-recorded. One microphone was attached close to the subject's mouth and another was placed in front of the interviewers. Two camcorders were positioned on tripods, facing each the subject from the right- and left angles. The subjects were confronted with some false statements about their whereabouts on a Saturday morning. They 
all denied such statements and tried to convince the interviewers that they were telling the truth.

Prior to conducting the study, we posed the following research questions:

Are there any give-away truth-telling cues? If yes, what are they?

Are there any differences in paralinguistic and linguistic cues between 'truth tellers' and 'liars' (based on personality traits)?

Are there any gender differences?

What role is played by cultural factors in denial strategies? What are the denial strategies used by the Japanese speakers from the Tohoku region?

What are the implications of our findings for forensic research?

There are a number of limitations to the present study. Firstly, with eleven subjects, the size of the study was too small to make any definite conclusions. Secondly, regarding the duration of the interviews, time constraints were imposed on the research team such that the interviews were limited to a maximum of 10 minutes per subject. Moreover, for this small-scale study, a semi-structured interview type was chosen and we focused on truth telling only. In future research, we will use a cognitive interview technique [16] that effectively discriminates truthful from false testimony and that consists of two components: (i) the initial, open-ended question: "Please describe everything you remember concerning the event" and (ii) the mnemonic prompt question designed to elicit more memory recall. This is a very effective cognitive device, whereby participants are asked to "take a moment and think about anything else they you may have seen, smelled, touched, tasted or felt during this experience. Once they you have done this, they would start at the beginning once more and tell everything they remember and include anything new they remember." [17]. According to Morgan III et al. [17], the mnemonic prompt of the cognitive interview resulted in a significantly higher unique word count in genuine, compared to deceptive eyewitnesses in their study.

Another limitation in the present study was the lack of stress exposure, unlike in genuine forensic situations. At present, one limiting factor concerns our understanding about the degree to which the findings from semi-structured interviews conducted in a university environment may be applied to real-world forensic cases that may involve highly stressful events. Research findings from psychobiological studies designed to assess the impact of realistic stress on human's show that such stress may result in significant alterations in cognition, perception, and memory [17-20].

\section{Results}

With reference to personality traits, Riggio, Tucker, and Widman [21] found that people who scored high in public selfconsciousness were poor liars-exhibiting e. g., less eye contact and more emotional reactions than those who scored low in public selfconsciousness. This implies that people's intentions to avoid displaying suspicious behaviour do not automatically mean that they will succeed in doing so.

Generally, extroverts show different and fewer cues to deception; they display fewer movements when they lie than when they are honest (= more movement when honest). Introverts have more disturbances in their speech during deception ([6], 16\% loc 1825)

\section{Questionnaire analysis}

The ratings for each statement done by the subjects were analysed by means of factor analysis, a statistic method used to detect positive and negative correlations of statements or questions such that highly correlated statements or questions that indicate some personality traits are grouped together. In our analysis, two groups of statements were found, each of which indicating traits of deceptiveness. The 16 statements in are listed in Table 1 below.

One group of positively correlated statements (Factor 1) includes statements $4,13,14,15$, and 16 , and these correlated negatively with statement 7 . Statements $13,14,15$, and 16 all indicate general traits of deceptiveness. This means that when a given subject rated high on statements $4,13,14,15$, and 16 , he/she rated low on statement 7 . Factor analysis indicated that those exhibiting traits of deceptiveness tend to care about what people think of them and have low self-esteem. Another group of positively correlated statements (Factor 2) includes statements 1, 3, 10 and 16, and these statements negatively correlate with statement 2 . This result indicates that someone who behaves deceptively to please others tends to be outgoing and does not show embarrassment much, but may feel nervous and uneasy in unfamiliar situations. See Appendix 2 for more details regarding this analysis.

Factor scores of factors 1 and 2 were calculated for each subject based on their statement ratings. Table 2 below shows the results. Negative values indicate that a person tends to be more truthful, and positive values indicate more deceptive traits. Consequently, the subjects were divided into two groups: 'truthful' (three males and two females) and 'deceptive' (two males and four females).

\section{Interview analysis}

During the course of each interview, the subjects' reactions and responses were video- and audio-recorded, and then analysed quantitatively and qualitatively. The analysis excluded responses during the small talk and those related to the fake question about the subjects' hometown. In the responses to false assumptions about the subjects' whereabouts expressed by the interviewers, some syntactic and semantic

1. I am a very communicative and outgoing person. I make friends easily and have no problem talking to strangers

2. I blush easily in embarrassing situations

3. When I'm not sure what is going on/l find myself in an unfamiliar situation I become nervous and cannot control my body movements easily (fidgeting, touching/scratching head, leg shifting, etc.)

4. I consider it to be important what other people think about me

5. I find it hard to deceive others because of a personality trait

6. I feel mental and physical discomfort when being put in spot light because of my questionable behaviour/when accused of something in front of others.

7. I have strong self-confidence/high self-esteem.

8. In general, I consider myself to be a risk-loving person.

9. I try to avoid risk as much as I can

10. I praise a new hairstyle of someone I know, even though it actually does not suit that person much.

11: I would make up a reason in order to avoid an uncomfortable meeting.

12: I keep a little secret to myself once in a while, even when someone directly asks about it.

13: I compliment important people, because they can do something for me.

14. I would lie for my own benefit.

15. I would lie for a friend's benefit.

16. I tell other people what they want to hear, even if it does not fully correspond to truth.

Table 1: 16 Statements in the questionnaire. 
Citation: Danielewicz-Betz A, Ogasawara N (2013) Truth Statements in Denial Context: A Study of Prosodic, Paralinguistic, and Discursive Cues in Japanese Speakers. J Forensic Res S11: 001. doi:10.4172/2157-7145.S11-001

Page 4 of 10

speech patterns were commonly observed, and some differences found between the truthful and deceptive groups. First, subjects in the truthful group denied the false assumptions more directly and firmly. When the interviewers said, for instance, "I saw you at the convenience store nearby the local train station last Saturday morning", they would deny this by saying something like "No, that's wrong," "I wasn't there," "Really, I DIDN'T stop by the station." Moreover, this group tended to utter complete sentences, without stopping mid-sentence. The subjects in the deceptive group, on the other hand, did not express direct denials as often as the truthful group; but rather, they would weaken their response by saying "I don't remember well... but maybe I didn't go there," "I think I wasn't there... maybe." Sometimes they would even go as far as to admit the false assumption by going along with the interviewers' suggestion and saying "Well, then... maybe I went out somewhere, I don't remember though," "If you say so, maybe then...," "You saw me? Really?... hmm...maybe. " In addition, compared with the truthful group, the deceptive group would drift away mid-sentence more often, as illustrated by these examples: "On Saturday... I went to a supermarket...only... that's why..." "Perhaps, I didn't step out... like...I guess...”. Table 3 below offers a summary of the response patterns.

Figure 1 displays the mean $\%$ in the subjects' denials when confronting false interviewer assumptions. An ANOVA was carried out with gender distinction (male, female) and personality traits (truthful, deceptive) as between-subjects factors. As the this figure shows, the truthful group denied significantly more often than the deceptive group $(\mathrm{F}(1,7)=16.0, p<0.01)$, and this tendency was common across genders, since neither significant gender effect nor interaction between the two factors were found $(p>0.1)$. On the other hand, as shown in Figure 2, the deceptive group admitted the false assumptions significantly more often than the truthful group $\operatorname{did}(\mathrm{F}(1,7)=7.5, p<0.03)$; and this tendency was more distinct among the male subjects than among the females subjects.

There were also more significant speech patterns observed for the deceptive group. Figure 3 below represents a graph of uncertain expressions. An ANOVA revealed that the male subjects in the deceptive group expressed uncertainty more often than the males in the truthful group $(\mathrm{F}(1,3)=29.3, p<0.02)$, but there was no significant difference among the female subjects $(p>0.1)$. Figure 4 represents a graph of incomplete utterances. The deceptive group tended to stop their utterances in the middle of a sentence more often than the truthful

\begin{tabular}{|c|c|c|}
\hline Subject & Factor 1 & Factor 2 \\
\hline Male 2 & -2.205 & -0.066 \\
\hline Male 4 & -0.706 & -1.630 \\
\hline Female 2 & -0.522 & 1.529 \\
\hline Male 1 & -0.474 & 0.338 \\
\hline Female 3 & -0.307 & -0.955 \\
\hline Female 1 & 0.338 & 0.860 \\
\hline Male 3 & 0.358 & 0.277 \\
\hline Female 4 & 0.435 & -0.370 \\
\hline Female 6 & 0.636 & 0.138 \\
\hline Male 5 & 0.896 & 1.161 \\
\hline Female 5 & 1.550 & -1.283 \\
\hline
\end{tabular}

Table 2: Factor scores (grey shaded subjects belong to the deceptive group).

\begin{tabular}{|c|c|c|c|c|c|}
\hline Subject & $\begin{array}{c}\text { \# of } \\
\text { utterances }\end{array}$ & $\begin{array}{c}\% \text { of } \\
\text { denials in } \\
\text { the whole } \\
\text { utterances }\end{array}$ & $\begin{array}{c}\text { \# of } \\
\text { admissions } \\
\text { of the false } \\
\text { assumptions }\end{array}$ & $\begin{array}{c}\text { \# of uncertain } \\
\text { expressions }\end{array}$ & $\begin{array}{c}\text { \# of } \\
\text { incomplete } \\
\text { sentences }\end{array}$ \\
\hline Male 2 & 29 & $58.6 \%$ & 0 & 0 & 0 \\
\hline Male 4 & 32 & $53.1 \%$ & 1 & 0 & 4 \\
\hline Female 2 & 32 & $46.9 \%$ & 0 & 0 & 1 \\
\hline Male 1 & 27 & $37.0 \%$ & 6 & 5 & 4 \\
\hline Female 3 & 21 & $42.9 \%$ & 0 & 4 & 1 \\
\hline Female 1 & 29 & $17.2 \%$ & 3 & 4 & 6 \\
\hline Male 3 & 28 & $10.7 \%$ & 5 & 13 & 3 \\
\hline Female 4 & 35 & $48.5 \%$ & 2 & 5 & 5 \\
\hline Female 6 & 38 & $23.6 \%$ & 3 & 8 & 6 \\
\hline Male 5 & 57 & $10.5 \%$ & 8 & 14 & 8 \\
\hline Female 5 & 20 & $30.0 \%$ & 2 & 4 & 5 \\
\hline
\end{tabular}

Table 3: Summary of response patterns (grey shaded subjects belong to the deceptive group).

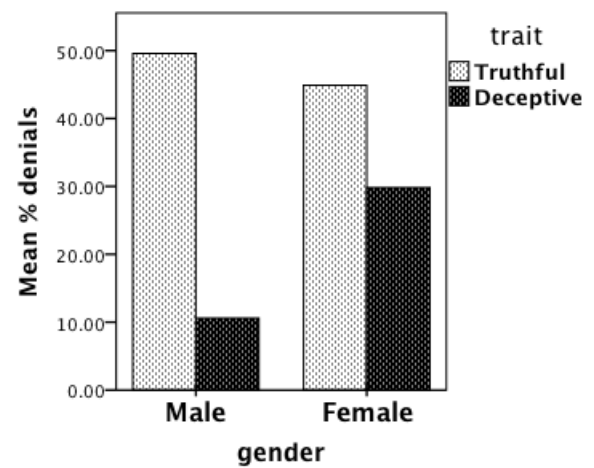

Figure 1: Mean \% denials in confrontation with false assumptions.

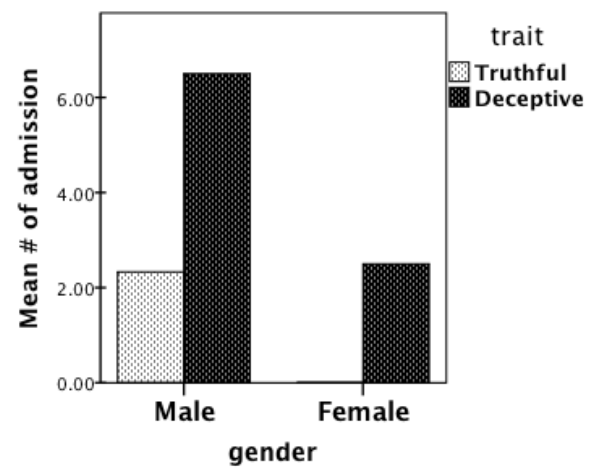

Figure 2: Mean number of times when false assumptions were admitted

group $(\mathrm{F}(1,7)=9.8, p<0.02)$, and this was commonly seen across both genders (gender effect $=p>0.1$ ).

Next, an acoustic analysis was carried out. Voice pitch $(\mathrm{Hz})$ and speech rate $(\mathrm{ms} / \mathrm{mora})$ for each utterance were measured. The Japanese language is a mora-timed language, with each mora considered to have almost the same duration. Thus, speech rate was calculated by 
dividing the duration of whole utterance by the number of morae in the utterance. Figure 5 shows the degree to which the subjects lowered their voice pitches in direct denial utterances such as "No, I was NOT there," as compared with other utterances. As it is shown, in all subjects except the males in the truthful group lowering of voice pitch was observed in direct denial utterances. The average pitch decrease in denials was $7.8 \mathrm{~Hz}$. Figure 6 illustrates the increase in utterance speed related to direct denials, as compared with non-denial utterances. Regardless of the personality traits and gender, all subjects spoke faster by 12.4 ms on average when making direct denials. In addition, this tendency was more apparent in the deceptive group than in the truthful group. Despite the fact that these acoustic characteristics are transparent in the graphs, the results were not confirmed by ANOVA. They were not statistically significant.

\section{Non-verbal behaviour}

Non-verbal behaviour has gained relative importance in credibility assessments. Several sources (police manuals, research findings, and real-life observations) indicate that non-verbal behaviour plays an important role in making veracity judgements. According to Inbau et al. [1], "as much as $70 \%$ of a message communicated between persons occurs at the non-verbal level".

Vrij and Granhag [22] list the non-verbal cues to deception found in published police interrogation manuals [1,23-27]. These include problem with eye contact, touching the nose, restless foot and leg movement, avoiding eye contact/direct gaze, frequent posture changes, grooming gestures, placing hands over mouth/eyes, rubbing the eyes,

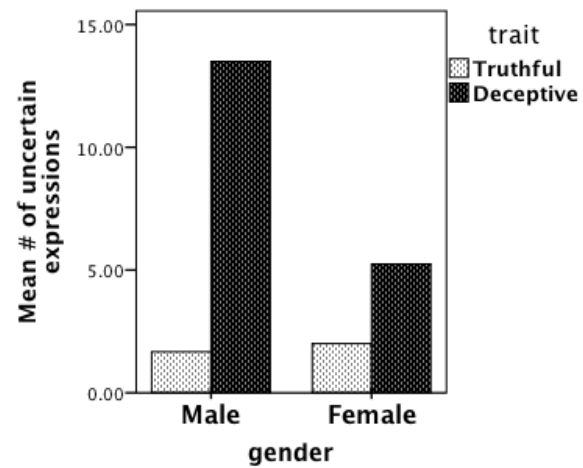

Figure 3: Mean number of uncertainty expressions in confrontation of false assumptions.

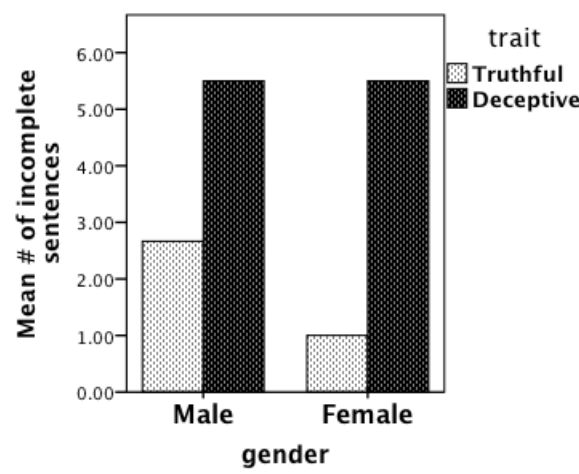

Figure 4: Mean number of incomplete utterances when dealing with false assumptions.

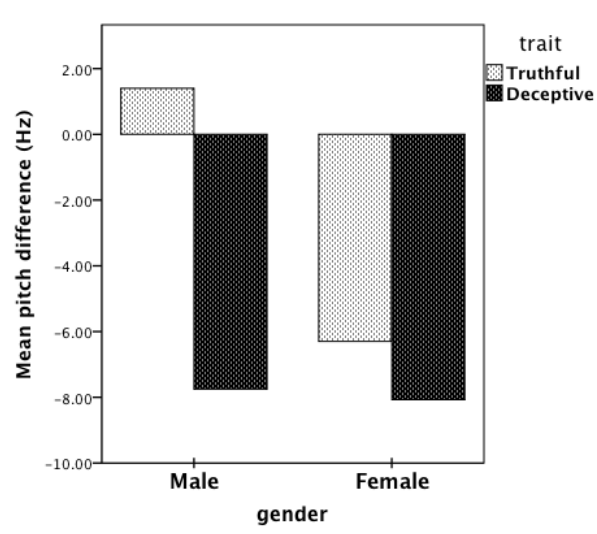

Figure 5: Mean lowered voice pitch in direct denial utterances compared with pitch in utterances other than direct denials.

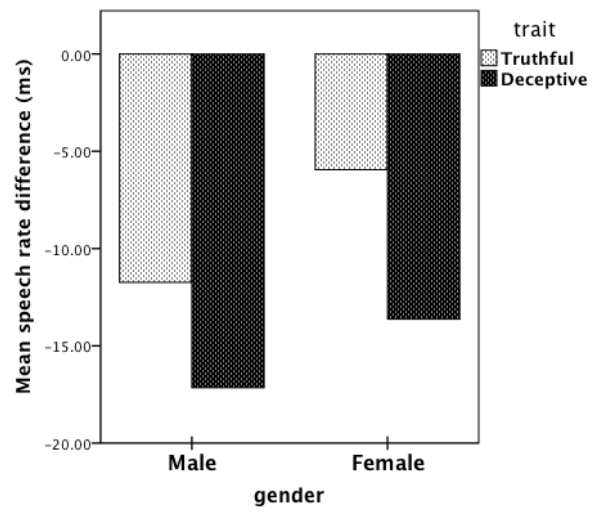

Figure 6: Mean speech rate differences in direct denial utterances compared with speech rate in utterances other than direct denials.

covering/rubbing the ears, restless behaviour, tapping of feet, fidgeting, excessive swallowing, shuffling the feet, picking lint from clothes, high frequency of blinking, moving the chair, abrupt and jerky behaviour, problem with fine motor coordination, cold and clammy hands, covering mouth with hands, as well as failure to maintain eye contact

Many of these cues were found in our subjects' non-verbal behaviour, whereby all of the subjects them were telling the truth when being recorded. Our body language analysis confirmed the reports from behaviour analysis interviews (reported in Vrij [6], 34\% Loc 3901) that truth tellers are more likely to shift posture; appear less helpful and show more signs of discomfort than liars. They are also more likely to lean forward, or establish eye contact, mirroring interviewers ' increase in movement.

Truth tellers are said to make more felt smiles than liars ([6], $14 \%$ loc 1624), a finding definitely confirmed in our study. Taking upon the distinction made earlier and based on the personality trait questionnaire, the following cues were found in 'truthful' and 'lying' subjects, respectively (Table 4).

\section{Pausing accompanied by body language}

The subjects typically exhibited frequent intra- and inter-turn pausing (or silent) behaviour, which shows that the Japanese truth tellers (at least from the Tohoku region) resort to extensive non-verbal behaviour when recalling past events, in an attempt to be as truthful as 
possible. We found out that pausing is not related to 'lying personality traits': it is shared by all the respondents.

There occurred frequent inter-turn silence after the interviewer's cue word "doyobi" (Saturday). The subjects repeated it up to six times per interview. All subjects used the echo question [do-yo-bi::?], followed by long intra-turn pauses $(2.5 \mathrm{~s}$ on average; and up to $5 \mathrm{~s}$ maximum duration). Moreover, excessive body language accompanied the silent 'doyobi recollection', whereby the subjects would look down or right/left, move their head up, move one hand up to the cheek, or would cover their nose and/or mouth, tilting the head and rolling the eyes upward.

\section{Head and eye movement: accessing memory}

According to Neuro-Linguistic Programming (NLP), automatic, unconscious eye movements, or "eye accessing cues," often accompany particular thought processes, and indicate the access and use of particular representational systems [28].

Each of the questions from the interview caused the subjects to access a memory or to mentally construct an experience. The effect noticed is referred to in neurological literature as 'lateral eyemovement'. Although it is impossible to tell whether someone is lying (but in the case of our study all the subjects were telling the truth) or what they are thinking about by watching their eyes, one can tell which 'sensory systems' they are accessing. In other words, eye movement is a guide to the thought process but not the content of a person's thoughts. Moreover, there will always be some exceptions to the patterns. But even somebody who is organised in a totally different way will be systematic in their eye movements. In Some people the patterns are reversed, typical in, but not exclusive to, many left-handed people (see Photos 1 and 2 for right/ left handed subjects accessing actual memory); others exhibit have a 'mixture' of traits [29].

Our truth telling subjects exhibited the following eye movements related to their recollections about the past event:

- Visually recalled memory: eyes go up and to the left (righthanded subjects) or to the right (the two left-handed subjects) - using actual memory in recollection;

- Audio recalled memory: eyes go to the left, looking to the ear (reversed direction for the two left-handed subjects) - accessing actual audio memory;

\begin{tabular}{|c|c|}
\hline 'TRUTHFUL’ & 'DECEITFUL' \\
\hline Less hand/finger movement in lap & $\begin{array}{c}\text { Looking rather self-confident, less } \\
\text { nervous }\end{array}$ \\
\hline Gesturing to reinforce a verbal point & $\begin{array}{l}\text { Speaking deliberately, less non-verbal } \\
\text { support by nodding or head shaking }\end{array}$ \\
\hline Nodding upward & Showing surprise \\
\hline $\begin{array}{l}\text { Appear a bit more nervous and } \\
\text { embarrassed (tense, shy smile) }\end{array}$ & $\begin{array}{l}\text { Laughter more natural, sounding less } \\
\text { embarrassed, smile more natural }\end{array}$ \\
\hline No much leg movement & Hand movement to chin when thinking \\
\hline Shoulder and head shaking & $\begin{array}{c}\text { Nodding and shaking of head less } \\
\text { vigorous }\end{array}$ \\
\hline Head/forehead scratching/massaging & $\begin{array}{l}\text { Eyes darting from one interviewer to } \\
\text { the other }\end{array}$ \\
\hline Quiet and/or nervous laughter; & Tongue rolling across lips \\
\hline More laughter in general & Swinging in chair \\
\hline More movement of hands to face & Fidgety fingers on thighs \\
\hline
\end{tabular}

Table 4: Body language in 'truthful' and 'deceitful' subjects.

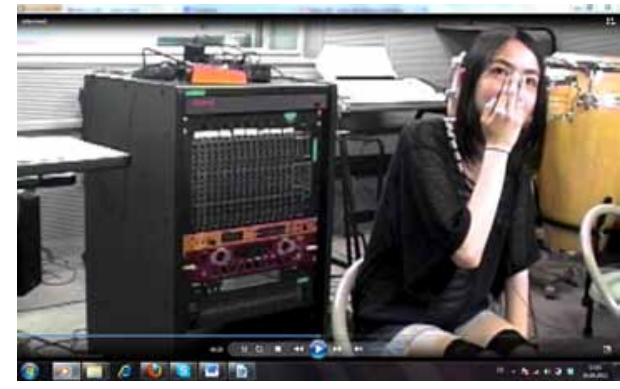

Photo 1: Accessing actual memory (right-handed subject).

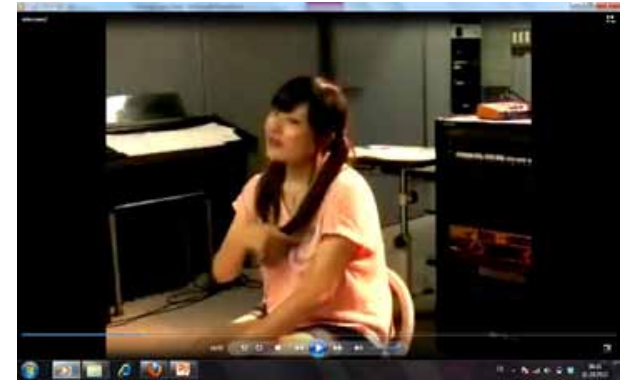

Photo 2: Accessing actual memory (left-handed subject)

- Actual emotional memory: eyes revert down and to the left (or right in the case of the two left-handed subjects): bringing back emotions experienced [30-32].

\section{Gender differences}

As mentioned before, researchers rarely report gender differences in their deception research ([6], 15\% loc 1727). In our study, the female subjects made an impression of trying to come across as likeable. They were more concerned with gaining acceptance of the interviewers and pleasing them than the male subjects. They would also laugh and giggle more (partly out of embarrassment). On the whole, they also exhibited more non-verbal signals supportive of the verbal statements (gesturing to illustrate a concept); more movement of hands to hair and clothes (touching and smoothing); hands to cheek or nose. Their mouths were open more frequently and the looked down.

The male subjects, on the other hand, tended to deny more directly and more frequently, without raising doubt. When pressed continually for answers to probing questions and statements like "Are you sure?" "I'm sure I saw you", they would finally give in and self-doubt or accommodate by saying something like "Maybe I don't remember". They also demonstrated less body movement when speaking, less necessity to support words with non-verbal signals; but, produced more hissing sounds, inhaling of air, bending forward and moving backward.

The typical and frequently used paralinguistic cues of truth telling observed in subjects are summarized in Table 5 below.

\section{Pragmatic analysis}

During the interviews the subjects exhibited a great deal of laughter-some of that due to jokes told by the interviewers, but also laughter due to embarrassment and feeling of discomfort. There was a noticeable impact of the interviewers; the subjects were trying to be 
courteous and polite, thus came across as self-conscious and not always getting to the point (avoiding being direct)

\section{Grice's Cooperative Principle-maxims of conversation}

Grice [33] identified three maxims of conversation, namely:

Maxims of Quantity: 1. "Make your contribution as informative as required." 2. "Don't make your contribution more informative than is required."

Maxims of Quality: Be truthful. 1. "Don't say what you believe to be false." 2. "Don't say what you lack adequate evidence for."

Maxim of Relation/Relevance: "Make your contribution relevant."

Maxims of Manner: 1. "Avoid obscurity of expression." 2. "Avoid ambiguity." 3. "Be brief “. 4. "Be orderly."

Grice also specified three ways in which one may not satisfy the maxims. One might just opt-out of one or more maxims, encounter a hopeless clash between two or more maxims, or flout ("blatantly fail to fulfil") one or more maxims. Unlike someone who is simply violating a maxim, someone who is flouting a maxim expects the listener to notice. Flouting a maxim is done in order to exploit it.

The maxims of relevance and of truthfulness, as well as the maxim of quantity-striving for brevity-were observed in all cases. The only maxim not always observed, due to the features of the Japanese conversation style and high-context cultural factors mentioned above, was the manner/clarity maxim. Violating, flouting a maxim, or opting out were not observed.

Besides cooperation, most interactions are governed by politeness, that is by what is considered a "polite social behaviour" within a certain culture. In accordance with positive politeness and to protect positive face of all the interlocutors [34] (see Leech 1983), the subjects offered accommodating responses, especially toward the end of the interview. Positive politeness means being complimentary and gracious to the addressee. Leech [34] defines politeness as a type of behaviour that allows the participants to engage in a social interaction in an atmosphere of relative harmony (harmony or "wa" 倭 is a key concept in Japanese culture). One of Leech's politeness maxims, namely the agreement maxim: minimize disagreement between self and other" maximize agreement between self and other, applied in particular to the interview responses of our respondents. So did the modesty maxim, i.e.: "that is: minimize praise of self; maximize dispraise of self."

So-called downgrades and hedges [35-36] expressing diluted

\begin{tabular}{|c|}
\hline Frequent hesitations and pausing when recollecting \\
\hline Inter-turn silence \\
\hline Tilting of head, eyes rolling up sideways \\
\hline Hand movement to mouth, face, nose, or hair \\
\hline Practically no movement/shuffling of feet \\
\hline Constant eye contact with interviewers \\
\hline Gestures supportive of verbal message \\
\hline Genuine smile and frequent laughter \\
\hline Lip rolling \\
\hline Expressive eyes \\
\hline Attentive listening \\
\hline
\end{tabular}

Table 5: Paralinguistic cues of truth telling. disagreement: "I think, maybe, I don't know, I'm sorry, but..."; or partial agreement, were also frequently were applied:

"I don't think so. Maybe the person looks like me. Hmm, maybe it's a mistake. Hmm, really? I forgot. Hmm, I think I was born in.... So I think I hadn't been there. I don't think I am wrong about my memory. Hmm, I think I didn't see any of my friends. I think I didn't go outside, but I am not sure. Maybe I went somewhere."

The term "downgrading" is used in conversation analysis, when the force of an utterance is downplayed in the way it is formulated as part of a response. Downgraded disagreements are a weaker form of disagreement [37-39].

The above excerpt also illustrates the process of 'back-channeling', typical for Japanese conversation. Backchanneling is a way of showing a speaker that you are following what they are saying and understand, often through interjections. It is a very important element in Japanese conversation so-called 相槌 (あいづち), which means a "brief response". It is very unnatural for someone to talk for a while without getting any response from their listeners. When a Japanese person is on the listening side during a conversation, he/she must say はい (“yes”), なるほど (“indeed”), そうですか (“really?”), or use similar backchanelling phrases signal to interlocutors that they are listening. All Japanese know that these phrases do not mean a 'yes', or imply any agreement.

Backchanneling was also carried out in the form of echo questions and answers, as illustrated below: (file 0214, female)

\section{Q: Were you at the train station?}

\section{A: The train station?}

Q: Were you at the train station?

\section{A: I don't think so. \\ Q1: I think you were there.}

\section{Q2: Last Saturday.}

\section{A: The train station...}

\section{Preferred responses in adjacency pairs}

Due to preference for agreement in Japanese conversation, in most cases, the preferred second parts of adjacency pairs were provided by the subjects. An adjacency pair is composed of two turns produced by different speakers which are placed adjacently and where the second utterance is identified as related to the first. Adjacency pairs include such exchanges as question/answer; complaint/denial; offer/acceptance; compliment/rejection; or challenge/rejection. Adjacency pairs typically have three characteristics, namely they consist of two utterances; the utterances are adjacent, that is the first immediately follows the second; with different speakers producing each utterance [40].

(File 0210, male)

Q: Did you do you part-time job on Saturday?

A: Last week, right?

Q: Yes, last week. Last Saturday.

\section{A: I remember I began my part-time job very early.}

Some second pair parts may be preferred and others may be dispreferred [41]. Levinson [42] names such common first pair parts of adjacency pairs as request, assessment, and blame, with the second pair 
parts being preferred or dispreferred, respectively: acceptance-refusal, agreement-disagreement, or denial-admission.

All questions in our data were followed by expected answers, like these ones:

Q: Definitely no? A: Yes.

Q: What were you doing last Saturday, about 10 o'clock, or 11 o'clock. A: I was sleeping.

Our discourse analysis of the interviews corroborates Hosoda's [13] findings, whereby he explains how Japanese speakers project disagreement and redesign their utterances to dilute or avoid disagreement - making use of grammatical resources and other discursive resources to project or anticipate other's actions in conversation. Agreements seem to occur quickly in a straightforward way, while disagreements are commonly delayed and mitigated.

Disagreements in Japanese conversation are often delayed through deployment of such elements as pauses, accounts, explanations, non-lexical perturbations (e.g. “ano::", “e::", “u::n”) and partial agreements, with the disagreement components commonly mitigated, e. g. by silence before a response to a question and then a pause before providing a reason for not doing something. Disagreement may also be delayed and expressed in an indirect manner: "Oh, is that right? I wonder if it is right."; or by an in-breath and a weak agreement "um" (yeah), accompanied by a hesitation, such as "ma:.:." (well...) ; or partial agreement "sou dakedo" (right, but) before stating one's opinion starting with "demo" (but). An utterance may be marked with hesitations, such as "hova" (you know), followed by in-breath and an account of one`s disagreement.

Linking the discursive cues, such as downgrades to the veracity assessment, let us cite Vrij [6,52\% - loc 5963 ] once again, who points out that lack of conviction or memory-being vague about certain elements in the statement ("I believe..", "I think...", "kind of...") or reporting that one cannot remember something is interpreted as suspicious by SCAN (scientific content analysis) users, but CBCA (criteria based content analysis) experts interpret lack of memory as a sign of truthfulness. This is confirmed by our observations of the subjects questioning their memory in the course of the interview, as can be illustrated by an excerpt from the transcripts of the audio recordings (see file 0214, female, literal translation):

\section{(...)}

Q: Were you in the store near to the train station?

A: I don't think so.

Q: Last week, it was raining. So I saw you in there.

A: (Thinking) Hmm.

Q: Were you there, right? (Repeatd) I have no idea about that.

A: I cannot remember that. Maybe I was there. I am not sure.

Q: Cannot remember? Hmm.

Q: I am sure that I saw you.

Q2: Saw her?

Q; I think I saw her.

Q2: You cannot remember that?

A: (Laugh)
Q: You were there, right? (Repeatd)

Q2: Please don't lie.

A: (Laugh)

Q: Were you there? No?

A: I think so.

Q: (inaudible)

Q: But I think I saw you. Maybe I looked wrong. But I don't think so.

A: (Laugh) maybe no. I think I didn't go outside last Saturday. I slept and then I did the part-time job.

Q: Hmm. You must be looking wrong.

Q: Did I look wrong? Were you there?

A: I went there sometimes.

Q: Did anyone say to you that you have a bad memory?

A: Yes. I am told this sometimes. (Laugh)

(...)

\section{Conclusions}

In this pioneer study, we investigated how Japanese people from the Tohoku region deal with false assumptions made by someone of higher rank and how they tell the truth in denial contexts. Although there are abundant studies on personality traits and studies on speech analysis, the majority of them have focused on lie detection and subjects have been mostly Caucasian English-speakers. Prior to the experiment, eleven subjects - undergraduate students (5 males and 6 females) - filled in a personality/behaviour questionnaire that was designed to detect their traits of lying. The subjects' responses were then statistically analysed using factor analysis [43] to determine whether a given subject exhibited lying traits or not. Among 11 subjects, 3 males and 2 females were assigned to the truthful group, with 2 males and 4 females classified as deceptive. Next, the subjects were individually interviewed by two senior students, who claimed false statements about the subjects' whereabouts on a Saturday morning. The subjects denied such statements, but the interviewers pretended not to believe them, insisting that the subjects were at the particular location, hence pressing for more information.

The qualitative questionnaire analysis and factor analysis indicated certain tendencies in the subjects' deceptive traits, allowing for a categorisation into two groups of subjects: truthful and deceptive. We analysed the subjects' responses and reactions during the individual interviews in which they were confronted with false assumptions and doubts on the part of the interviewers.

The analysis revealed some significant linguistic and paralinguistic characteristics of speech in truth-telling denial context. First, grammatical, lexical, and pragmatic characteristics were found. The deceptive group uttered more incomplete sentences, used expressions of uncertainty, and tended to eventually admit the false assumptions made, instead of denying them directly. On the other hand, the truthful group denied directly and uttered complete sentences more often than the deceptive group. These results seem to reflect the subjects' personalities. The factor analysis found that subjects who exhibited deceptive traits care about what others think of them and tend to have low self-esteem. Hence, it was possible that the deceptive group hesitated to deny the false assumption facing the interviewers; in an 
attempt to accommodate their speech and behaviour in order to satisfy the interviewers. Contrastively, the truthful subjects were relatively less self-conscious, whereby what others thought about them was not so important to them. So they were more certain in responding to the false assumptions denying more directly and firmly.

The acoustic analysis of voice pitch and speech rate led to some prosodic tendencies. In general, direct denials were uttered faster and at a lower pitch than non-denial utterances, and this tendency was more apparent among the subjects in the deceptive group. These results are related to some previous acoustic studies on lying and persuasive speech. In the study of Streeter et al. [44], for example, the subjects' voice pitch increased more when lying than when telling the truth. Our acoustic analysis found the Miller effect: since all the subjects were telling the truth in denial context, they also tended to lower their voice. On the perception side, Miller et al. [45] found that faster speech enhanced persuasion in perception; and Apple et al. [46] reported that speakers with high-pitched voices were perceived less truthful, whereas slow talkers were judged less truthful and less persuasive. These previous studies suggest a possibility that if such perception patterns (i. e. high pitched voice and slow speech rate influencing perception of utterances as less truthful) are universal, the subjects in the current study consciously or unconsciously used the strategy of lowering their voices and speaking faster so that their denials are judged as more convincing by the interviewers. However, this is just an assumption at this point. A future perception study of truth-telling would be required to validate this further.

The body language analysis turned out inconclusive: we demonstrated that the cues identified in deceit by previous studies might occur in truth-telling as well. Some tendencies but no significant gender differences were observed in paralinguistic behaviour (e. g. for the female subjects to employ more non-verbal signals supportive of verbal messages). We may therefore conclude that there exists no single behavioural or verbal cue uniquely related to deception, i. e. there is no give-away cue ([6], 72\% loc 8254). The same is valid for truth telling. Therefore, it is preferable to make veracity assessments on the basis of multiple cues [47-50], which implies looking for clusters of cues and paying attention to non-verbal and verbal cues simultaneously.

To conclude, in a future study we will attempt to overcome the limitations of the present research mentioned above, aiming at recruitment of a larger number of subjects, using cognitive interview techniques and both truth and deceit scenarios in a simulated forensic setting. We also intend to carry out a cross-cultural comparison, involving Japanese, Vietnamese, and Chinese subjects.

\section{Implications and Future Research}

Having demonstrated how easily Japanese subjects may switch from telling the truth to toning down definite assertions, downright to inventing information just to please interviewers and maintain harmony, one of the future forensic directions will be to investigate the implication of the pragmatics of Japanese conversation style (embedded in the Japanese culture and its values) in the coercive interview context. In Japan, the conviction rate is more than $99 \%$, often based on confessions. A confession has been called 'the king of evidence':

"They kept on at me to confess. So I thought, it's probably better to make the false confession and that's why I did it. "(http://news.bbc. co.uk/2/hi/asia-pacific/7063316.stm).
Recently several cases related to false confessions and false convictions, a result of intimidating interrogation methods used by the Japanese police have been reported in the media:

"When I first started saying I was innocent the intimidation and the pressure on me grew stronger." They kept saying, 'Your family is giving up on you, they're very disappointed in you. 'They kept repeating it over and over. (...) And this also involves many, many hours of repeated questions and sometimes sleeps deprivation, and where the detainee is given the impression he would only be released once he confesses. (...) In that kind of system, what's of great concern is the confessions extracted could even be used to sentence a person to death. "(http:// news. bbc.co.uk/2/hi/8290767.stm).

It has been proven that authorities, such as police investigators, take advantage of high power relations to implicate a (perhaps innocent) person in a crime. In the United States, the Supreme Court in Miranda v. Alabama set down the requirement that, prior to the arrest or interrogation of a suspect in a crime, that person must be told that they have the right to remain silent, the right to legal counsel, and the right to be told that anything they say can be used in court against them. Instances of the application of this requirement serve well as an illustration of how speech acts performed by police officers may lead to the apparent 'consensual' nature of searches, how questioning can be interpreted as coercive, and how the relationship between authority figures and a suspect/defendant is asymmetric. Consider the following examples, discussed in Solan and Tiersma [51] which on the semantic level cannot be interpreted as directives, yet pragmatically speaking, given the authoritarian context, appear precisely as that:

Does the trunk open?

You don't mind if we look in your trunk, do you?

Why don't you put your hands behind your back, all right?

The level of coerciveness increases in 'requests' such as:

Would you mind if I took a look around here?

Well, then, you don't mind if I look around in the car, do you, or would you?

The police usually lack the authority to make promises such as "We'll go easy on you if you confess", yet this is implied in their "requests" to comply. The problem is, as Solan and Tiersma [51] point out, that people who are stopped by the police tend to interpret ostensible requests as commands or orders, yet, by in contrast, their own indirect wishes to get a lawyer often go unnoticed (for example, "Maybe I should talk to a lawyer"). In any case, the asymmetric nature of the relationship between authority figures (the police) and the defendant-who may be disadvantaged in some way-can result in a text (such as a record of interview, video or audio recording, or written statement) which is considerably at variance with what the suspect would have said had he/she been given the opportunity to make a statement in a non-coercive or less threatening environment. This leads to the conclusion that despite the necessity of strong contextual reliance in the interpretation of speech acts, courts may habitually use out-of-context inferences and entailments to reach decisions and that suspect interviews should be video-recorded for future evidence [52].

\section{References}

1. Inbau F, Reid J, Buckley J, Jayne B (2011) Criminal interrogation and confessions. Jones and Bartlett Learning.

2. O`Sullivan M (2005) Emotional intelligence and deception detection: Why most 
Citation: Danielewicz-Betz A, Ogasawara N (2013) Truth Statements in Denial Context: A Study of Prosodic, Paralinguistic, and Discursive Cues in Japanese Speakers. J Forensic Res S11: 001. doi:10.4172/2157-7145.S11-001

Page 10 of 10

people can't "read" others, but a few can. In Riggio, R E and Feldman, R S (Eds). Applications of nonverbal communication (pp. 215-253). Mahwah, NJ, Erlbaum.

3. O'Sullivan M (2007) Unicorns or Tiger Woods: are lie detection experts myths or rarities? A response to on lie detection "wizards" by Bond and Uysal. Law Hum Behav 31: 117-123.

4. Bond CF Jr, DePaulo BM (2006) Accuracy of deception judgments. Pers Soc Psychol Rev 10: 214-234.

5. Mann SA, Vrij A, Fisher RP, Robinson M (2008) See no lies, hear no lies: Differences in discrimination accuracy and response bias when watching or listening to police suspect interviews. Applied Cognitive Psychology 22: 1062 1071.

6. Vrij A (2008) Detecting Lies and Deceit: Pitfalls and Opportunities. (2ndedn), John Wiley and Sons, Ltd., Kindle edition, Chichester, England, United Kingdom.

7. Omura M, Ukitani S (2005) Tohokujin wa donna seikakuka. (What personality Tohoku people have.) Japan Society of Personality Psychology 14: 79-80.

8. Sitton SC, Griffin ST (1981) Detection of deception from clients' eye contact patterns. Journal of Counseling Psychology 28: 269-271.

9. Vrij A, Winkel F W (1991) Cultural patterns in Dutch and Surinam nonverbal behaviuor: An analysis of simulated police/citizen encounters. J Nonverbal Behav 3: 169-184.

10. Lawrence AA (2010) Societal individualism predicts prevalence of nonhomosexual orientation in male-to-female transsexualism. Arch Sex Behav 39: $573-583$

11. Hammond A (2001) Think Japan. Aimai: The Importance of Ambiguity.

12. Yamakuse $Y$ (2009) likaesanai Nihonjin (Japanese don`t talk back.) IBC Publishing Co, Tokyo.

13. Hosoda Y (2009) Diluting Disagreement in Japanese Conversation Departmental Bulletin Paper 169: 87-117.

14. Murai K (2011) They Say Yes, They Mean No.

15. Abeler J, Becker A,Falk A (2012) Truth-telling-A representative assessment. Mimeo, University of Oxford.

16. Geiselman RE, Fisher RP (1997) Ten years of cognitive interviewing. In: Payne, $\mathrm{D}$ and Conrad, $\mathrm{F}$ (Eds) Intersections in basic and applied memory research. Mahwah, NJ: Lawrence Erlbaum: 291-310.

17. Morgan CA 3rd, Colwell K, Hazlett GA (2011) Efficacy of forensic statement analysis in distinguishing truthful from deceptive eyewitness accounts of highly stressful events. J Forensic Sci 56: 1227-1234.

18. Morgan CA 3rd, Rasmusson AM, Winters B, Hauger RL, Morgan J, et al. (2003) Trauma exposure rather than posttraumatic stress disorder is associated with reduced baseline plasma neuropeptide-Y levels. Biol Psychiatry 54: 10871091.

19. Morgan CA 3rd, Wang S, Rasmusson A, Hazlett G, Anderson G, et al. (2001) Relationship among plasma cortisol, catecholamines, neuropeptide $Y$, and human performance during exposure to uncontrollable stress. Psychosom Med 63: 412-422.

20. Morgan CA 3rd, Doran A, Steffian G, Hazlett G, Southwick SM (2006) Stressinduced deficits in working memory and visuo-constructive abilities in Special Operations soldiers. Biol Psychiatry 60: 722-729.

21. Riggio RE, Tucker J, Widaman KF (1987) Verbal and nonverbal cues as mediators of deception ability. J Nonverbal Behav 11: 126-145.

22. Vrij A, Granhag PA (2007) Interviewing to detect deception. In Christianson, S A (Ed), Offenders' memories of violent crimes (pp. 279-304). John Wiley and Sons, Ltd., Chichester, England.

23. Gordon NJ, Fleisher WL (2002) Effective interviewing and interrogation techniques. (3rdedn.), NewYork: Academic Press.

24. Macdonald, J M, and Michaud, D L (1992) Criminal interrogation. Apache Press, Denver, CO
25. Rabon D, Chapman T (1992) Interviewing and interrogation. Carolina Academic Press, Durham, NC.

26. Yeschke CL (1997) The art of investigative interviewing. (2ndedn.), ButterworthHeinemann, Newton, Massachusetts.

27. Zulawski DE, Wicklander DE (2001) Practical aspects of interview and interrogation. CRC Publishing.

28. Dilts R (1998) Eye movements and NLP.

29. Farlam J (2011) Eye accessing cues.

30. Grinder J, DeLozier J, Bandler R (1977) Patterns of the Hypnotic Techniques of Milton $\mathrm{H}$. and Erickson, MD, Meta Publications, Cupertino, $\mathrm{Ca}$.

31. Bandler R, Grinder J (1979) Frogs into princes: Neuro linguistic programming. Real People Press, Moab, Utah.

32. Dilts R, Grinder J (1980) Neuro-Linguistic Programming, The Structure of Subjective Experience, Cupertino.

33. Grice P (1975) Logic and conversation. In Cole P, Morgan JL (Eds) (pp. 41-58) Speech Acts. Academic Press, New York.

34. Leech GN (1983) Principles of pragmatics London: Longman.

35. Lakoff G (1973) Hedges: A study in meaning criteria and the logic of fuzzy concepts. Journal of philosophical logic 2: 458-508.

36. Brown P, Levinson SC (1987) Politeness: Some universals in language usage Cambridge University Press.

37. Pomerantz AM (1984) Giving a source or basis: The practice in conversation of telling 'how I know'. Journal of pragmatics 8: 607-625.

38. Blum-Kulka S, House J, Kasper G (1989) Cross-cultural pragmatics: Requests and apologies, Ablex Pub.

39. Hutchby I, Wooffitt R (2008) Conversation analysi, Polity Press.

40. Thornbury S, Slade D (2006) Conversation: From Description to Pedagogy, Cambridge University Press.

41. Paltridge $B$ (2007) Making sense of discourse analysis, Gold Coast

42. Levinson SC (1983) Pragmatics (Cambridge textbooks in linguistics) Cambridge University Press.

43. Levinson SC (1983) Pragmatics (Cambridge textbooks in linguistics) Cambridge University Press.

44. Russell DW (2002) In search of underlying dimensions: The use (and abuse) of factor analysis in Personality and Social Psychology Bulletin. Personality and social psychology bulletin 28: 1629-1646.

45. Streeter LA, Krauss RM, Geller V, Olson C, Apple W (1977) Pitch changes during attempted deception. J Pers Soc Psychol 35: 345-350.

46. Miller N, Murayama G, Beaber RJ, Valone K (1976) Speed of speech and persuasion. Journal of Personality and Social Psychology 34: 615-624.

47. Apple W, Streeter LA, Krauss RM (1979) Effects of Pitch and speech rate on personal attributions. Journal of Personality and Social Psychology 37: 715727

48. Ekman P, O Sullivan M, Friesen WV, Scherer KR (1991) Invited article: Face voice, and body in detecting deceit. J Nonverbal Behav 15: 125-135.

49. Vrij A, Akehurst L, Soukara S, Bull R (2004) Let Me Inform You How to Tell a Convincing Story: CBCA and Reality Monitoring Scores as a Function of Age, Coaching, and Deception. Canadian Journal of Behavioural Science 36: 113126.

50. Vrij A, Edward K, Roberts KP, Bull R (2000) Detecting deceit via analysis of verbal and nonverbal behaviour. J Nonverbal Behav 24: 239-263.

51. Vrij A, Mann S (2004) Detecting deception: The benefit of looking at a combination of behavioral, auditory and speech content related cues in a systematic manner. Group Decision and Negotiation 13: 61-79.

52. Solan LM, Tiersma PM (2005) Speaking of Crime: The Language of Criminal Justice, The University of Chicago Press.

53. Danielewicz-Betz, A (2012) The Role of Forensic Linguistics in Crime Investigation. In Littlejohn, A and Rao Mehta, S (Eds) Language Studies: Stretching the Boundaries (pp. 93-108). Cambridge Scholars Publishing, Newcastle upon Tyne, UK. 